\title{
LOS ISÓPODOS (CRUSTACEA, PERACARIDA) ASOCIADOS AL SISTEMA ARRECIFAL BAJOS DE SISAL Y PUERTO PROGRESO, YUCATÁN, MÉXICO
}

\author{
Manuel Ortiz ${ }^{1}$, Norma Berenice Cruz-Cano ${ }^{1}$, IgnacioWinfield ${ }^{1}$, \\ Sergio Cházaro-Olvera ${ }^{1}$ y Miguel Lozano-Aburto ${ }^{2}$
}

\begin{abstract}
${ }^{1}$ Laboratorio de Crustáceos, Facultad de Estudios Superiores Iztacala, Universidad Nacional Autónoma de México, Av. de los Barrios No 1, Los Reyes Iztacala, Tlalnepantla, Estado de México, México. ortiztouzet@yahoo.com; nbiocc7@gmail.com; ignacioc@unam.mx; chazaro@campus.iztacala.unam.mx

${ }^{2}$ Laboratorio de Invertebrados marinos, Instituto de Ciencias Marinas y Pesquerías, Universidad veracruzana, Boca del Río, Veracruz, México.gorgoniamx@hotmail.com
\end{abstract}

\section{RESUMEN}

En este trabajo se analizan la composición faunística, la distribución y la abundancia de los isópodos asociados a diferentes sustratos del Sistema Arrecifal Bajos de Sisal y Puerto Progreso. Las recolectas fueron realizadas manualmente, con el empleo de SCUBA, en los meses de mayo y junio del 2012, en 16 sitios de muestreo, entre 1 y $20 \mathrm{~m}$ de profundidad. Se obtuvieron organismos asociados a camas de algas, esponjas, pedacería de coral, madera, sedimento, boyas y pilotes. Se cuantificaron 3,604 isópodos pertenecientes a 5 subórdenes, 5 superfamilias, 18 familias, 28 géneros y 49 especies. La familia con la mayor riqueza específica fue Anthuridae (ocho especies). La especie Carpias algicola presentó la abundancia mayor (1,693 individuos). El sitio con abundancia mayor fue Bajo de Diez (1,066 individuos), siendo Madagascar el que presentó la riqueza específica mayor. Cuatro especies constituyen registros nuevos para el Golfo de México, mientras que 14 especies presentan ampliaciones del ámbito geográfico, hasta el sureste del golfo.

Palabras clave: Isopoda, lista de especies, distribución, SE Golfo de México.

Title: The isopods (Crustacea, Peracarida) associated to Bajos de Sisal coral reefs and Puerto Progreso, Yucatán, México.

\section{ABSTRACT}

The abundance, distribution and faunal composition of the isopods associated to different substrates of Bajos de Sisal Coral Reef System and Puerto Progreso were analyzed. Hand samples by using SCUBA were obtained from May to June 2012, in 16 localities, at depths of 1-20 m. Organisms were collected from buoys, pilots, sediment, wood, coral rubble, sponges and algae beds. A total of 3,604 isopods belonging to five suborders, five superfamilies, 18 families, 28 genera and 49 species were identified. The Anthuridae was the family with the highest species richness (eigth species). The most abundant species was Carpiasalgicola (1,693 specimens). Algae was the most important substrata during this study. The locality with the highest abundance was Bajo de Diez (1,066 specimens), and Madagascar was the site with the highest species richness. Four species were first recorded for the Gulf of Mexico, while 14 species represent geographical extensions towards the southeast of the Gulf.

Keywords: Isopoda, check list, distribution, SE Gulf of Mexico. 


\section{INTRODUCCIÓN}

El estudio de la diversidad biológica y abundancia en los sistemas arrecifales está basado frecuentemente en los componentes macroscópicos como corales, briozoos, moluscos, cangrejos y peces, (Kensley, 1998). En estos ambientes se encuentran numerosos organismos asociados a diferentes sustratos, entre los cuales están los isópodos.

La guía de Kensley y Schotte (1989), para la identificación de los isópodos del Golfo de México y el Mar Caribe constituye una herramienta básica para identificar especies, aún en la actualidad. Posteriormente, Schotte et al. (2009) elaboraron un listado de todos los isópodos del Golfo de México que contiene 169 especies, distribuidas en 6 subórdenes y 37 familias. Más tarde, Ortiz et al. (2013) analizaron la abundancia y distribución de los isópodos en el Área Natural Protegida Tuxpan-Lobos, en donde reportaron 32 especies, agrupadas en 11 familias y 20 géneros; de los cuales 11 eran nuevos registros y 16 presentaban ampliación del ámbito geográfico.

A pesar de que se han publicado numerosos trabajos sobre los isópodos del Golfo de México, ninguno se ha referido al Sistema Arrecifal Bajos de Sisal.El presente estudio pretende contribuir al conocimiento de este importante grupo de crustáceos peracáridos, en la zona mencionada.

\section{OBJETIVO}

- Analizar la diversidad, la abundancia y la distribución de los isópodos asociados a diferentes sustratos de los Sistemas arrecifales Bajos de Sisal (SABS) y de Puerto Progreso (PPr).

\section{MATERIALES Y MÉTODOS}

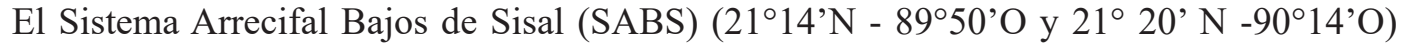
(Fig. 1) se encuentra localizado al oeste de la Península de Yucatán, presentando una temperatura media anual de $26^{\circ} \mathrm{C}$, con una precipitación media anual de 400-1100 mm. El clima de la región es semiseco y cálido subhúmedo con lluvias en verano (Instituto Nacional de Estadística y Geografía, 2009). Dadas sus características, se les considera como arrecifes de tipo plataforma (Zarco-Perelló et al., 2013).

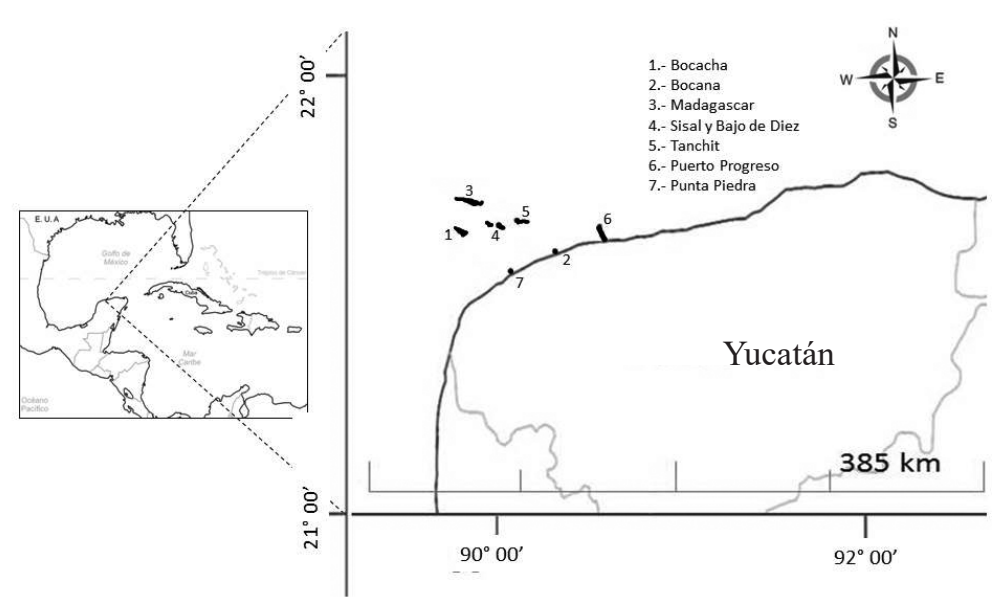

Figura 1. Ubicación geográfica de las estaciones estudiadas en el Sistema arrecifal Bajos de Sisal y Puerto Progreso. 
Los especímenes fueron recolectados en los meses de mayo y junio del 2012, en 16 sitios de muestreo entre 1 y $20 \mathrm{~m}$ de profundidad. Se colectaron isópodos sobre camas de algas, esponjas, pedacería de coral, madera, sedimento, boyas y pilotes, de forma manual y con ayuda de equipo autónomo SCUBA. Posteriormente, todos los sustratos fueron colocados en bolsas y envases de plástico. Una vez en superficie se agregaron gotas de una solución de alcohol-formaldehído (1:1) para excitar y obtener los crustáceos asociados. Todas las muestras fueron procesadas con ayuda de un tamiz con abertura de malla de $500 \mu \mathrm{m}$, para ser fijadas con alcohol al $70 \% \mathrm{y}$ almacenadas en frascos debidamente etiquetados.

En el Laboratorio de Crustáceos, de la Facultad de Estudios Superiores Iztacala-UNAM, los isópodos fueron separados para su identificación con ayuda de microscopía óptica y estereoscópica hasta el nivel más bajo posible, de acuerdo a las claves específicas de Schultz (1969), Kensley y Schotte (1989) y Kensley (1997).

El listado taxonómico se realizó de acuerdo a la clasificación propuesta por Ahyong et al., (2011). Posteriormente, mediante la revisión bibliográfica correspondiente, se determinaron los registros nuevos y las ampliaciones del ámbito geográfico, de acuerdo a la división del Golfo de México propuesta por Felder et al., (2009). Los especímenes identificados fueron depositados en la Colección Nacional de Crustáceos (CNCR) del Instituto de Biología de la UNAM (2831128369; 28796-28821).

\section{RESULTADOS}

Se cuantificó un total de 3604 organismos, pertenecientes a cinco subórdenes, cinco superfamilias, 18 familias, 27 géneros y 49 especies. Los subórdenes con el mayor número de familias fueron: Cymothoida, con ocho y Asellota, con seis. Las familias con la riqueza específica mayor fueron: Anthuridae (ocho especies), y Corallanidae (cinco especies), siguiéndole en importancia Janiridae, Santiidae, Stenetriidae y Leptanthuridae con tres especies cada una. Las familias restantes estuvieron representadas por una sola especie.

El sitio que presentó la mayor abundancia de individuos fue Bajo de Diez con 1,066 (equivalente al 30\%), seguido de Sisal con 586 (16\%). Los sitios con abundancia menor fueron Bocana con 226 (6\%) y Puerto Progreso con 240 (7\%) (Fig. 2).

La familia más representada fue Janiridae, con 2,126 individuos (equivalente al 59\%), seguida por Sphaeromatidae con 680 (19\%) e Idoteidae con 169 (5\%). Las familias menos representadas fueron Munnidae, con $4(0.11 \%)$, Paranthuridae e Hyssuridae, con 3 cada una $(0.08 \%)$ (Fig. 3).

Los sustratos con la abundancia mayor de organismos fueron los de macroalgas, con 2,015 individuos (equivalente al 56\%) y las esponjas, con 874 (24\%). Además, las macroalgas presentaron la riqueza mayor de familias (14), seguido de pedacería de coral (13), esponjas (seis), madera (cinco) y por último, balanos y ascidias con tres. Las familias con mayor frecuencia en la mayoría de los sustratos, excepto en el sedimento, fueron Corallanidae y Janiridae. Las familias que sólo se presentaron en un sustrato fueron: Aegidae, Ancinidae, Arcturidae y Paranthuridae

\section{DISCUSIÓN}

Registros nuevos. El presente trabajo contribuye con cuatro registros nuevos de isópodos para el Golfo de México: Amakusanthura cf. paramagnifica Müller, 1992, Mesanthura punctillata Kensley, 1982, Accalathura setosa Kensley, 1984 y Metacirolana agaricicola Kensley, 1984. 


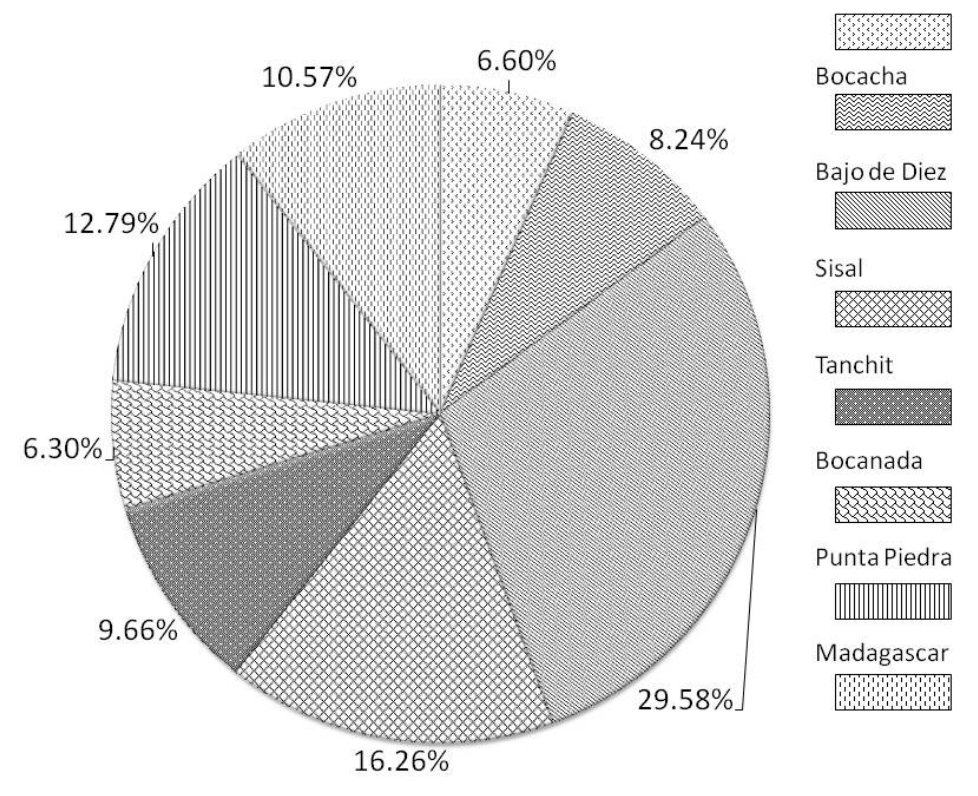

Puerto Progreso

Figura 2. Abundancia de isópodos por sitios de colecta.

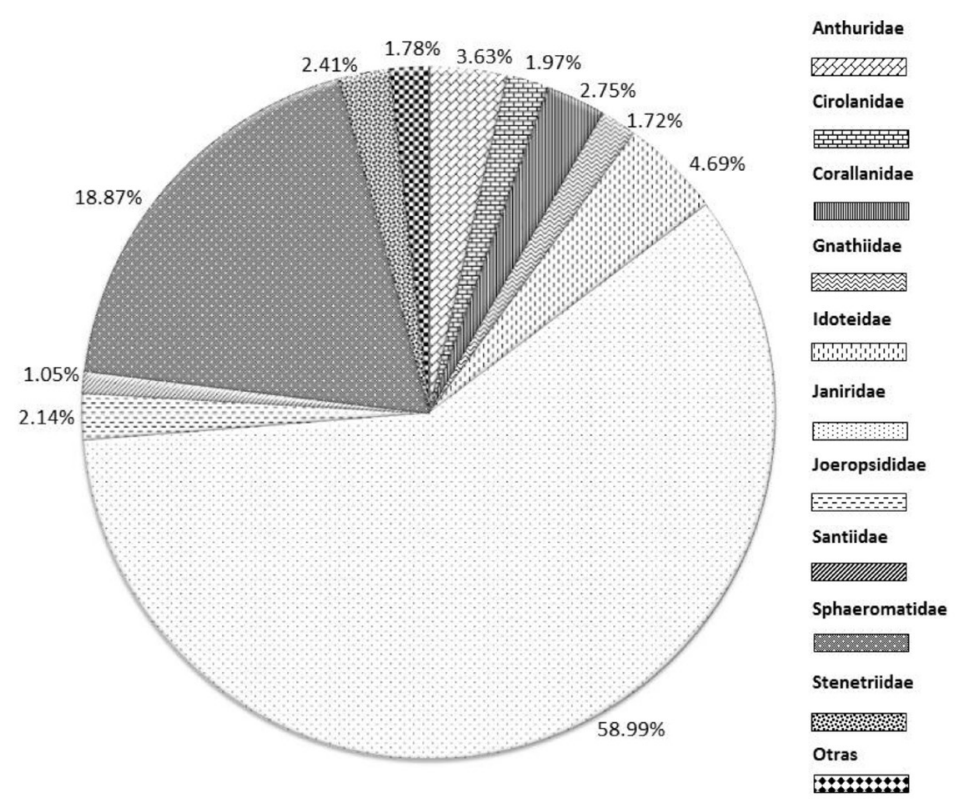

Figura 3. Abundancia de las familias de isópodos representadas en la zona de estudio.

Ampliaciones del ámbito geográfico. Además, 14 especies ampliaron su ámbito geográfico hasta el sector sureste del golfo de México, de acuerdo a la división propuesta por Felder et al. (2009), donde se encuentra el SABS (Tabla 1).

Composición faunistica. Las familias Anthuridae, Aegidae, Cirolanidae, Corallanidae, Excorallanidae, Sphaeromatidae y Joeropsididae han sido reportadas con anterioridad para el Banco de Campeche (Escobar-Briones et al., 2008; Escobar-Briones y Jiménez-Guadarrama, 2010). 


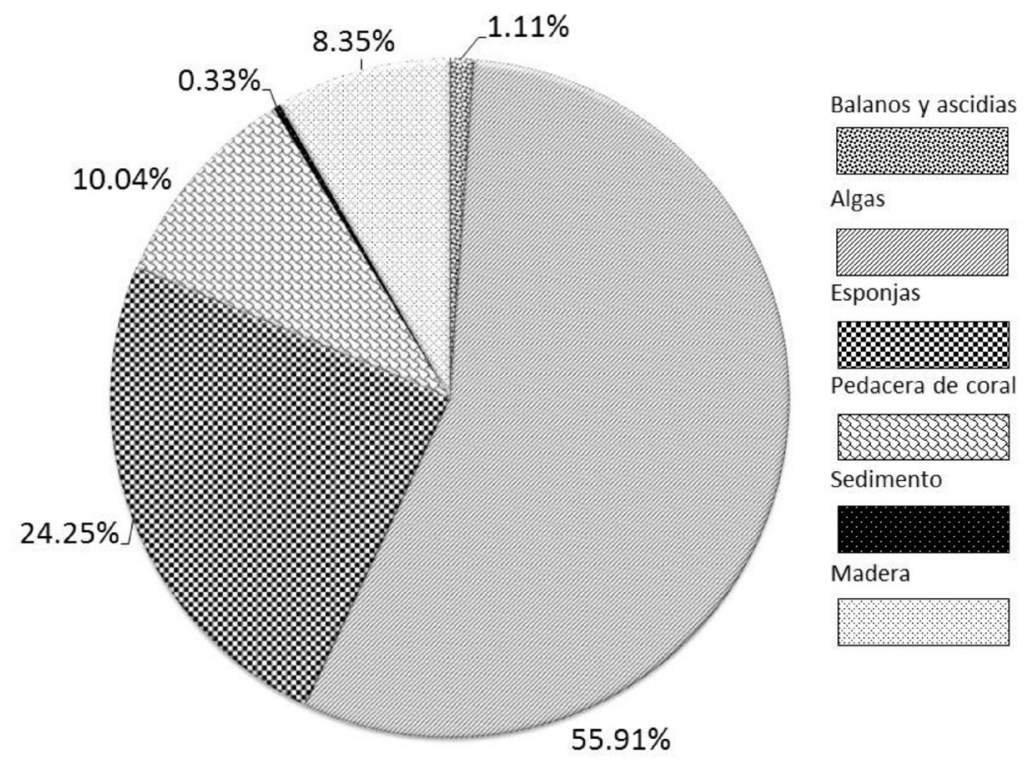

Figura 4. Representación gráfica de la abundancia de isópodos por tipo de sustrato.

La mayor riqueza específica ocurrió con la familia Anthuridae, con ocho especies (Tabla 1). Kensley (1998) documentó que en los arrecifes de coral estos isópodos están muy bien representados, debido a que probablemente su cuerpo cilíndrico se ajusta perfectamente a las grietas, huecos, tubos y conchas que se encuentran en ese tipo de sustrato. La mayoría de estos organismos se encontraron asociados a algas, pedacería de coral y esponjas, lo que concuerda con lo reportado por Kensley (1997), Poore y Bruce (2012) y Ortiz et al., (2013).

La familia Janiridae ha sido la más abundante en este trabajo, 2,126 individuos (Fig. 3). Se trata de una familia cosmopolita y típica de aguas someras, con ciertos representantes en el mar profundo (Doti y Wilson, 2010). A dicha familia pertenece Carpias algicola uno de los isópodos más abundantes en aguas tropicales someras y en nuestro estudio. Registrada con anterioridad en el sector suroeste del Golfo de México (Kensley y Schotte, 2002; Winfield y Ortiz, 2011; Ortiz et al., 2013).

Aunque las familias Anthuridae, Paranthuridae, Leptanthuridae e Hyssuridae son generalmente las más diversas y abundantes en los arrecifes de todo el mundo (Kensley, 1997; Kensley, 1998; Poore y Bruce, 2012), no tuvieron una gran representación en los resultados de este trabajo.

El sustrato que presentó la abundancia mayor de organismos fue el de las macroalgas (2015 especímenes, pertenecientes a 14 familias), con el 56\% de la abundancia total (Fig. 4). Las algas, especialmente abundantes en el SABS (Zarco- Perelló et al., 2013), constituyen el hábitat idóneo para una gran diversidad de invertebrados marinos, entre los que se destacan los crustáceos peracáridos (Hinojosa et al., 2007). Son utilizadas además, para alimentación, reproducción, crianza y refugio (Winfieldet et al., 2007). También, son sitios con una alta heterogeneidad ambiental y complejidad arquitectónica (Boström y Bonsdorf, 2000).

Finalmente, debe destacarse que el SABS ha resultado más rico en especies que los sistemas Tuxpan-Lobos y Veracruzano estudiados por (Ortiz et al., 2013; Winfield et al., 2010). 


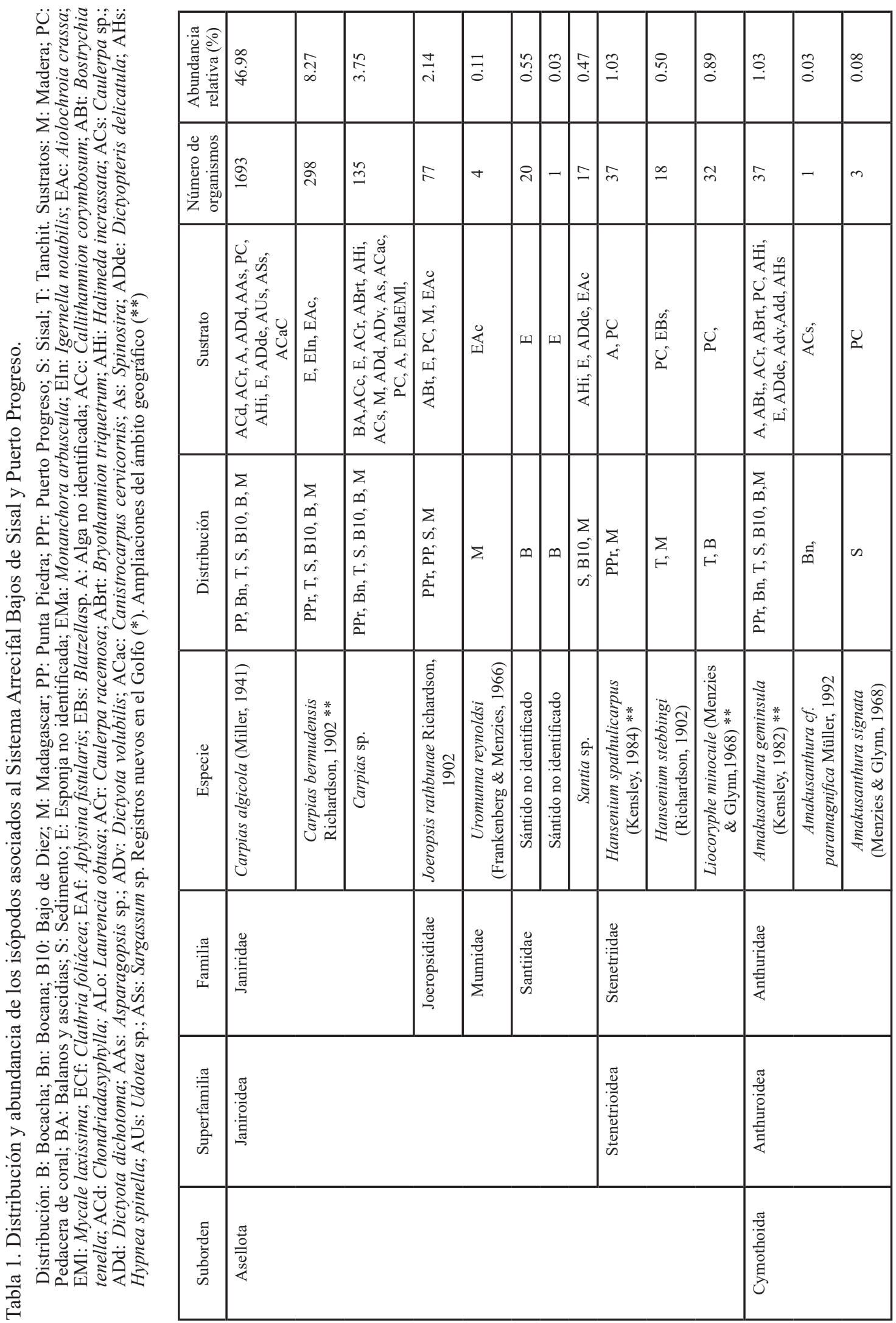




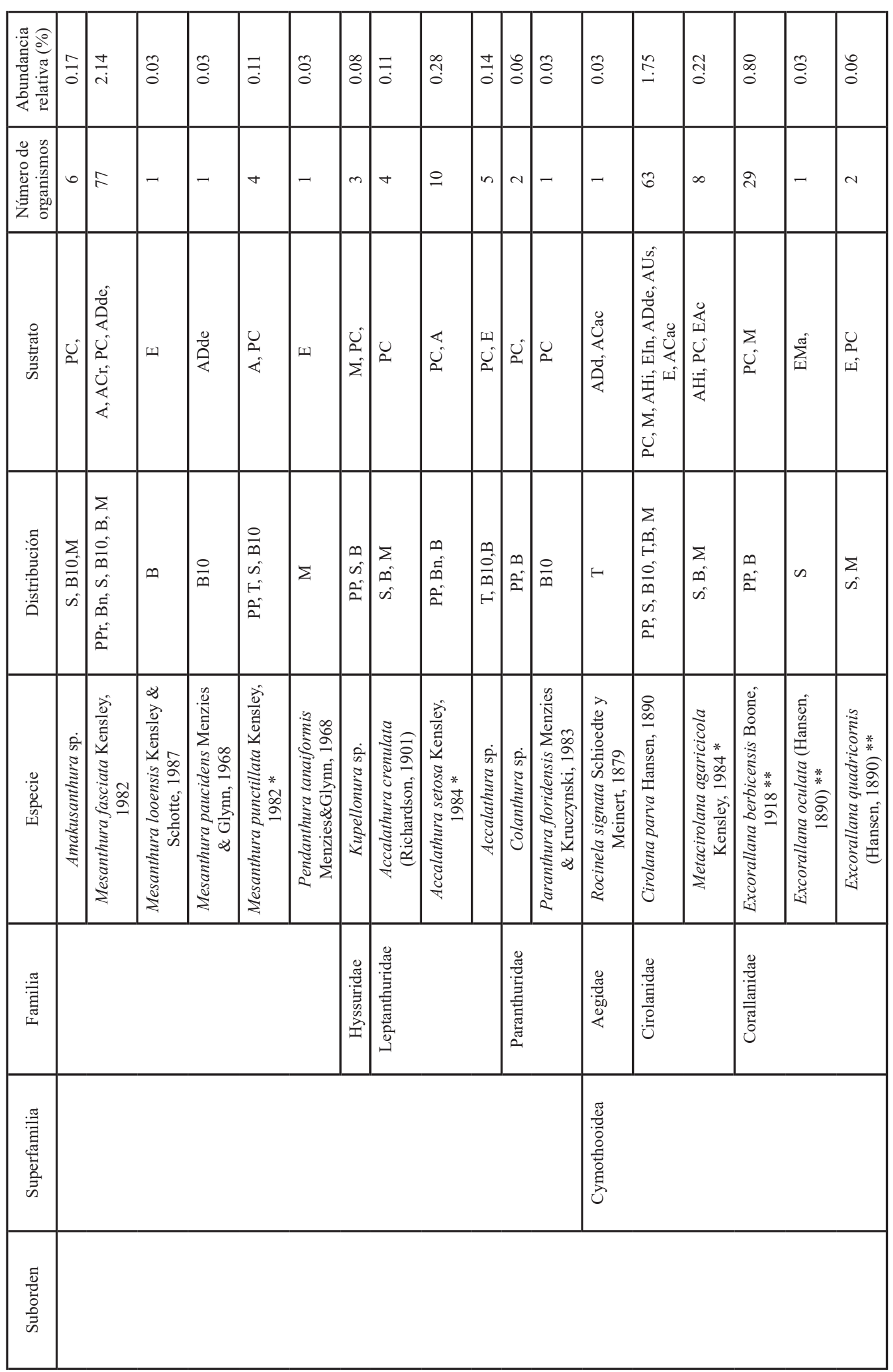




\begin{tabular}{|c|c|c|c|c|c|c|c|c|c|c|c|c|c|c|c|c|c|}
\hline 造 & $\stackrel{\infty}{\infty}$ & 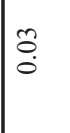 & $\stackrel{\infty}{\stackrel{\infty}{-}}$ & $\begin{array}{l}0 \\
0 \\
0\end{array}$ & 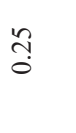 & $\begin{array}{l}m \\
\stackrel{0}{0}\end{array}$ & 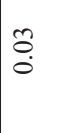 & $\stackrel{0}{\circ}$ & $\begin{array}{l}2 \\
0 \\
0\end{array}$ & $\stackrel{\infty}{\stackrel{\infty}{-}}$ & $\mid \begin{array}{l}0 \\
\dot{0}\end{array}$ & $\hat{\varrho}$ & $\stackrel{7}{0}$ & $\frac{a}{0}$ & $\vec{F}$ & $\mid \begin{array}{l}\infty \\
0 \\
0\end{array}$ & $\frac{2}{0}$ \\
\hline 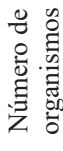 & 8 & - & ले & $\tilde{\lambda}$ & $a$ & $\approx$ & - & $\sim$ & $\stackrel{\infty}{\sim}$ & t & -7 & ন্ & 0 & $n$ & $\stackrel{2}{2}$ & $m$ & 12 \\
\hline 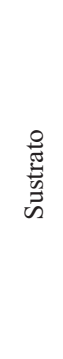 & 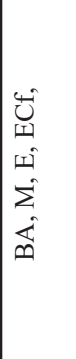 & $\ll$ & ¿ & 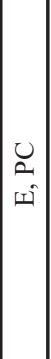 & 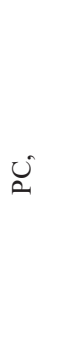 & $n$ & $\varangle$ & 吕 & 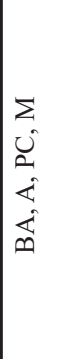 & 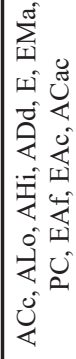 & 디 & 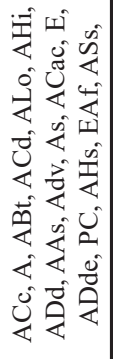 & 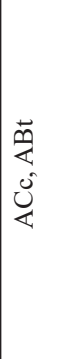 & 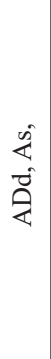 & 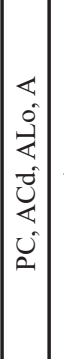 & $\varangle$ & 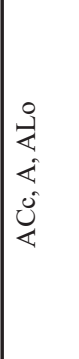 \\
\hline 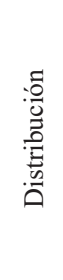 & 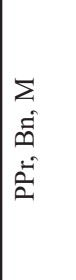 & $\mid \Sigma$ & $\begin{array}{l}\Sigma \\
\infty \\
\dot{n} \\
\mapsto \\
\mapsto\end{array}$ & $\mid \begin{array}{l}\Sigma \\
n \\
n \\
n \\
-1\end{array}$ & 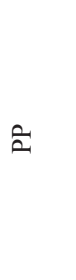 & 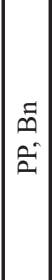 & 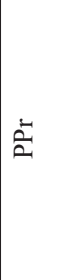 & 帘 & 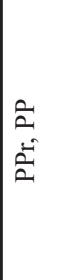 & 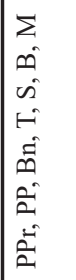 & is & 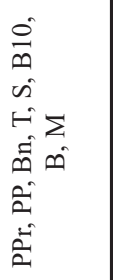 & $\overrightarrow{2}$ & $\mapsto$ & $\approx$ & $\infty$ & $\begin{array}{l}\overrightarrow{\mathrm{E}} \\
\stackrel{\mathrm{a}}{\mathrm{a}}\end{array}$ \\
\hline 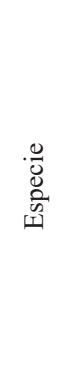 & 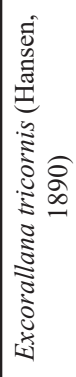 & 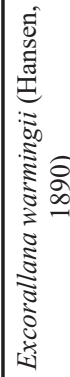 & 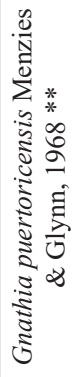 & 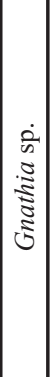 & 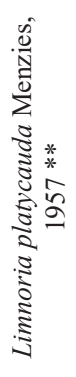 & 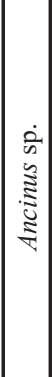 & 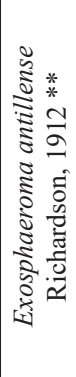 & 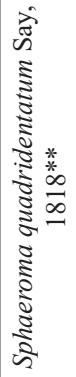 & 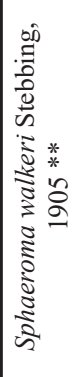 & 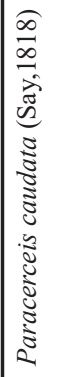 & 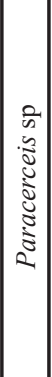 & 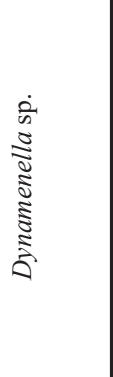 & 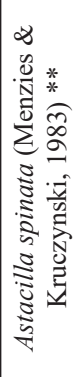 & 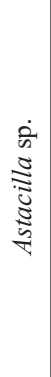 & 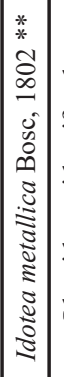 & 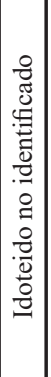 & 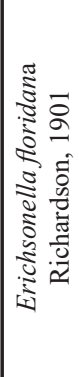 \\
\hline 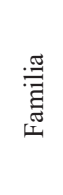 & & & $\begin{array}{l}\text { 莺 } \\
\text { 壳 } \\
\text { Jే }\end{array}$ & & 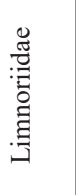 & 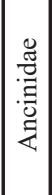 & 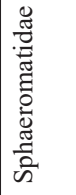 & & & & & & 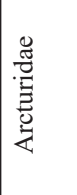 & & 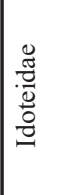 & & \\
\hline 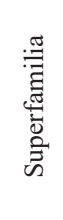 & & & & & & 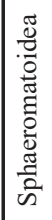 & & & & & & & & & & & \\
\hline 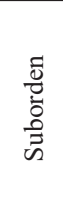 & & & & & 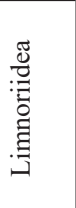 & 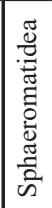 & & & & & & & 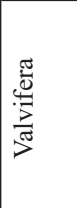 & & & & \\
\hline
\end{tabular}




\section{AGRADECIMIENTOS}

A la Dirección General de Asuntos de Personal Académico (DGAPA), particularmente al Programa de Apoyo a Proyectos de Innovación y Mejoramiento de la Enseñanza (PAPIME), número PE207311 (UNAM) y PAPIIT-IN229011 (UNAM) por el apoyo financiero otorgado.

A las autoridades de la CONAPESCA-DGOPA (SAGARPA) por los permisos otorgados para la colecta científica en Sisal y Puerto Progreso. Así mismo a la Administración Portuaria Integral Puerto Progreso, Yucatán, por las facilidades para colectar organismos en zona federal.

\section{LITERATURA CITADA}

Ahyong, S. T., J. K. Lowry, M. Alonso, R. N. Bamber, G. A. Boxshall, P. Castro, S. Gerken, G. S. Karaman, J. W. Goy, D. S. Jones, K. Meland, D. C. Rogers y J. Svavarsson. 2011. Subphylum Crustacea, Brünnich, 1772. En Z.- Q Zhang (Ed.) Animal biodiversity: An outline of higher-level classification and survey of taxonomic richness. Zootaxa. 3148:165-191.

Boström, C. y E. Bonsdorff. 2000. Zoobenthic community establishment and hábitat complexity - The importance of seagrass shoot-density, morphology and physical disturbance for faunal recruitment. Marine Ecology Progress Series. 205:123-138.

Doti, B. L. y G. D. F Wilson. 2010. The genera Carpias Richardson, IaniropsisSarsandJanaira Moreira \&Pires (Isopoda: Asellota: Janiridae) from Australia, with description of three new species. Zootaxa. 2625: 1-39.

Escobar-Briones, E. G., A. Gaytán-Caballero y P. Legendre. 2008. Epibenthicmegacrustaceans from the continental margin, slope and abyssal plain of the Southwestern Gulf of Mexico: Factors responsable for variability in species composition and diversity. Deep-Sea Research II. 55: 2667-2678.

Escobar-Briones, E. G y E. L. Jiménez-Guadarrama. 2010. Macrocrustáceos (Peracarida, Decapoda) de fondos carbonatados del sector occidental del banco de Campeche en el sur del golfo de México. Revista Mexicana de Biodiversidad. 81: S63-S72.

Felder, D. L., D. K. Camp y J. W. Tunell Jr. 2009. An Introduction to Gulf of Mexico Biodiversity Assesment. En The Gulf of Mexico, origins, water and biota. Vol. I. D. L. Felder y D. K. Camp (eds).Texas A\&M University Press. United States of America. p. 1-13.

Hinojosa, I., E. González, P. Ugalde, N. Valdivia, E. Macaya y M. Thiel. 2007. Distribución y abundancia de macroalgas flotando a la deriva y su fauna peracárida asociada en los canales de la XI Región, Chile. Ciencia y Tecnología Marina. 30 (2): 37-50.

Instituto Nacional de Estadísticas y Geografía (INEGI). 2009. Prontuario de información geográfica municipal de los Estados Unidos Mexicanos. Hunucmá, Yucatán. Clave geoestadística 31038. 2009. (En línea, consultado el 28 de Agosto del 2012) www.inegi. org.mx

Kensley, B. 1997. Identification, distribution, and aspects of the biology of ten anthuridean isopods species from the shallow continental shelf of the U.S. Gulf and East Coast. Gulf Research Reports. 9 (4): 277-302. 
Kensley, B. 1998. Estimates of species diversity of free-living marine isopod crustaceans in coral reefs.Coral reefs. 17:83-88.

Kensley, B. y M. Schotte. 1989. Guide to the marine isopod crustaceans of the Caribbean. Smithsonian Institution Press, Washington, D.C. 308 pp.

Kensley, B. y M. Schotte. 2002. New species and records of Asellota from the Indian Ocean (Crustacea: Peracarida: Isopoda). Journal of Natural History, 36: 1421-1461.

Ortiz, M., I. Winfield, S. Cházaro-Olvera, B. López-Del Río y M. Á. Lozano-Aburto. 2013. Isópodos (Crustacea: Peracarida) del área natural protegida arrecife Tuxpan-Lobos, Veracruz, México: lista de especies y registros nuevos. Novitates Caribaea 6: 63-75.

Poore, G. C. y N. L. Bruce. 2012. Global Diversity of Marine Isopods (Except Asellota and Crustacean Symbionts). Plus One 7:8-15.

Schotte, M., J. C. Markahm, y G. D. F. Wilson. 2009. Isopoda (Crustacea) of the Gulf of Mexico. en The Gulf of Mexico, Origins, Waters and Biota. Vol. I. D. L. Felder y D. K. Camp (eds). Texas A\&M University Press. United States of America. p. 973-986.

Schultz, G. 1969. How to know the marine isopod crustaceans.Wno C. Brown Company Publishers.U.S.A. 359.World Register of Marine Species (WoRMS).2013. Consultado el $1^{\text {ro }}$ de Octubre del 2013 en : http://www.marinespecies.org/.

Winfield, I., S. Cházaro-Olvera, y F. Álvarez, 2007. ¿Controla la biomasa de pastos marinos la densidad de los peracáridos (Crustacea : Peracarida) en lagunas tropicales?. Revista de Biología Tropical. Vol 55(1):43-53.

Winfield I., S. Cházaro-Olvera, G. Horta-Puga, M. Lozano-Aburto y V. Arenas-Fuentes. 2010. Macroscrustáceos incrustantes en el Parque Nacional Sistema Arrecifal Veracruzano: biodiversidad, abundancia y distribución. Revista mexicana de Biodiversidad. 80: S165-S175.

Winfield, A. I. y Ortiz, M. 2011. Crustáceos con bolsa incubadora (Crustacea: Malacostraca: Peracarida). En La biodiversidad en Veracruz: Estudio de estado. Volumen II. Comisión Nacional para el conocimiento y Uso de la biodiversidad, Gobierno del Estado de Veracruz, Universidad Veracruzana, Instituto de Ecología A.C. México. p 277-286.

Zarco-Perelló S., M. Mascaró, R.Garza-Pérez y N. Simoes. 2013. Topography and coral community of the Sisal Reefs, Campeche Bank, Yucatán, México. Hidrobiológica 23(1): 28-41.

[Recibido: 03 de abril, 2014. Aceptado para publicación: 09 de septiembre, 2014] 